\title{
Descriptive statistics
}

Table 1. Predicted distance under full (F-RCHB) and incomplete (I-RCHB) vision condition (Mean \pm SD).

\begin{tabular}{|l|l|l|l|l|l|l|}
\hline & \multicolumn{7}{|c|}{ Predicted distance $[\mathbf{m}]$} \\
Condition & $\mathbf{1 . 5} \mathbf{~ m}$ & $\mathbf{2 . 0} \mathbf{~ m}$ & $\mathbf{2 . 5} \mathbf{~ m}$ & $\mathbf{3 . 0} \mathbf{~ m}$ & $\mathbf{3 . 5} \mathbf{~ m}$ & $\mathbf{4 . 0} \mathbf{~ m}$ \\
\hline I-RCHB & $2.61 \pm 0.95$ & $2.88 \pm 1.11$ & $3.15 \pm 1.06$ & $3.15 \pm 1.21$ & $3.07 \pm 1.06$ & $3.05 \pm 0.99$ \\
\hline F-RCHB & $2.47 \pm 1.05$ & $2.89 \pm 0.94$ & $3.66 \pm 0.99$ & $3.83 \pm 1.11$ & $4.36 \pm 1.02$ & $4.62 \pm 1.00$ \\
\hline
\end{tabular}

Table 2. Follow-up analyses (Wilcoxon test with Bonferroni correction) of the interaction of putting distance and vision condition for predicted putting distance.

\begin{tabular}{|l|l|l|l|l|l|l|}
\hline & $\mathbf{1 . 5} \mathbf{~ m}$ & $\mathbf{2 . 0} \mathbf{~ m}$ & $\mathbf{2 . 5} \mathbf{~ m}$ & $\mathbf{3 . 0} \mathbf{~ m}$ & $\mathbf{3 . 5} \mathbf{~ m}$ & $\mathbf{4 . 0} \mathbf{~ m}$ \\
\hline $\mathrm{N}$ & 20 & 20 & 20 & 20 & 20 & 20 \\
\hline $\mathrm{Z}$ & -1.755 & -.485 & -3.099 & -3.192 & -3.920 & -3.920 \\
\hline $2 \mathrm{p}$ & .079 & .627 & $.002^{*}$ & $.001^{*}$ & $<.001^{*}$ & $<.001^{*}$ \\
\hline
\end{tabular}

* Significant after Bonferroni correction. Level of significance $p<.008 \overline{3}$.

Table 3. Constant error of predicted distance under full (F-RCHB) and incomplete (I-RCHB) vision condition (Mean \pm SD).

\begin{tabular}{|l|l|l|l|l|l|l|}
\hline & \multicolumn{6}{|c|}{ Constant error of predicted distance $[\mathbf{m}]$} \\
Condition & $\mathbf{1 . 5} \mathbf{~ m}$ & $\mathbf{2 . 0} \mathbf{~ m}$ & $\mathbf{2 . 5} \mathbf{~ m}$ & $\mathbf{3 . 0} \mathbf{~ m}$ & $\mathbf{3 . 5} \mathbf{~ m}$ & $\mathbf{4 . 0} \mathbf{~ m}$ \\
\hline I-RCHB & $1.11 \pm 0.95$ & $0.88 \pm 1.11$ & $0.65 \pm 1.06$ & $0.15 \pm 1.21$ & $-0.43 \pm 1.06$ & $-0.95 \pm 0.99$ \\
\hline F-RCHB & $0.97 \pm 1.05$ & $0.89 \pm 0.94$ & $1.16 \pm 0.99$ & $0.83 \pm 1.11$ & $0.86 \pm 1.02$ & $0.62 \pm 1.00$ \\
\hline
\end{tabular}

Table 4. Follow-up analyses (Wilcoxon test with Bonferroni correction) of the interaction of putting distance and vision condition for constant error.

\begin{tabular}{|l|l|l|l|l|l|l|}
\hline & $\mathbf{1 . 5} \mathbf{~ m}$ & $\mathbf{2 . 0} \mathbf{~ m}$ & $\mathbf{2 . 5} \mathbf{~ m}$ & $\mathbf{3 . 0} \mathbf{~ m}$ & $\mathbf{3 . 5} \mathbf{~ m}$ & $\mathbf{4 . 0} \mathbf{~ m}$ \\
\hline $\mathrm{N}$ & 20 & 20 & 20 & 20 & 20 & 20 \\
\hline $\mathrm{Z}$ & -1.755 & -.485 & -3.099 & -3.192 & -3.920 & -3.920 \\
\hline $2 \mathrm{p}$ & .079 & .627 & $.002^{*}$ & $.001^{*}$ & $<.001^{*}$ & $<.001^{*}$ \\
\hline
\end{tabular}

* Significant after Bonferroni correction. Level of significance $p<.008 \overline{3}$. 
Table 5. Variable error of predicted distance under full (F-RCHB) and incomplete (I-RCHB) vision condition (Mean \pm SD).

\begin{tabular}{|l|l|l|l|l|l|l|}
\hline & \multicolumn{6}{|c|}{ Variable error predicted distance $[\mathbf{m}]$} \\
Condition & $\mathbf{1 . 5} \mathbf{~ m}$ & $\mathbf{2 . 0} \mathbf{~ m}$ & $\mathbf{2 . 5} \mathbf{~ m}$ & $\mathbf{3 . 0} \mathbf{~ m}$ & $\mathbf{3 . 5} \mathbf{~ m}$ & $\mathbf{4 . 0} \mathbf{~ m}$ \\
\hline I-RCHB & $0.58 \pm 0.32$ & $0.64 \pm 0.41$ & $0.58 \pm 0.41$ & $0.72 \pm 0.41$ & $0.64 \pm 0.46$ & $0.65 \pm 0.35$ \\
\hline F-RCHB & $0.65 \pm 0.52$ & $0.63 \pm 0.36$ & $0.59 \pm 0.27$ & $0.63 \pm 0.34$ & $0.57 \pm 0.31$ & $0.51 \pm 0.30$ \\
\hline
\end{tabular}

Table 6. Results of the two-factor ANOVA with repeated measures (6 distances; 2 vision conditions) for the variable error of the predicted putt length. Corrected by Greenhouse-Geisser $\epsilon$.

\begin{tabular}{|l|l|l|l|l|}
\hline Factor & df1 & df2 & F & p \\
\hline Vision condition & 1.00 & 19.00 & 1.09 & .310 \\
\hline Distance & 3.56 & 67.70 & .454 & .748 \\
\hline Vision condition $x$ distance & 3.78 & 71.74 & .808 & .581 \\
\hline
\end{tabular}

Table 7. Absolute error of predicted distance under full (F-RCHB) and incomplete (I-RCHB) vision condition (Mean \pm SD).

\begin{tabular}{|l|l|l|l|l|l|l|}
\hline & \multicolumn{6}{|c|}{ Absolute error predicted distance $[\mathbf{m}]$} \\
Condition & $\mathbf{1 . 5} \mathbf{~ m}$ & $\mathbf{2 . 0} \mathbf{~ m}$ & $\mathbf{2 . 5} \mathbf{~ m}$ & $\mathbf{3 . 0} \mathbf{~ m}$ & $\mathbf{3 . 5} \mathbf{~ m}$ & $\mathbf{4 . 0} \mathbf{~ m}$ \\
\hline I-RCHB & $1.21 \pm 0.81$ & $1.12 \pm 0.87$ & $0.99 \pm 0.75$ & $0.99 \pm 0.69$ & $0.91 \pm 0.69$ & $1.14 \pm 0.75$ \\
\hline F-RCHB & $1.08 \pm 0.92$ & $1.00 \pm 0.82$ & $1.27 \pm 0.85$ & $1.12 \pm 0.80$ & $1.11 \pm 0.73$ & $0.99 \pm 0.63$ \\
\hline
\end{tabular}

Table 8. Results of the two-factor ANOVA with repeated measures (6 distances; 2 vision conditions) for the absolute error of the predicted putt length. Corrected by Greenhouse-Geisser $\epsilon$.

\begin{tabular}{|l|l|l|l|l|}
\hline Factor & df1 & df2 & F & p \\
\hline Vision condition & 1.00 & 19.00 & .174 & .681 \\
\hline Distance & 2.30 & 43.78 & .356 & .732 \\
\hline Vision condition $x$ distance & 2.50 & 47.45 & 2.05 & .129 \\
\hline
\end{tabular}

Table 9. Confidene of prediction under full (F-RCHB) and incomplete (I-RCHB) vision condition (Mean \pm SD).

\begin{tabular}{|l|l|l|l|l|l|l|}
\hline & \multicolumn{6}{|c|}{ Confidence $[\mathbf{1 - 5}]$} \\
Condition & $\mathbf{1 . 5} \mathbf{~ m}$ & $\mathbf{2 . 0} \mathbf{~ m}$ & $\mathbf{2 . 5} \mathbf{~ m}$ & $\mathbf{3 . 0} \mathbf{~ m}$ & $\mathbf{3 . 5} \mathbf{~ m}$ & $\mathbf{4 . 0} \mathbf{~ m}$ \\
\hline I-RCHB & $2.70 \pm 0.85$ & $2.63 \pm 0.83$ & $2.56 \pm 0.84$ & $2.66 \pm 0.94$ & $2.68 \pm 0.83$ & $2.57 \pm 0.80$ \\
\hline F-RCHB & $3.19 \pm 0.84$ & $3.09 \pm 0.81$ & $3.18 \pm 0.76$ & $3.24 \pm 0.77$ & $3.27 \pm 0.81$ & $3.40 \pm 0.72$ \\
\hline Difference & 0.49 & 0.46 & 0.62 & 0.58 & 0.60 & 0.85 \\
\hline
\end{tabular}

Table 10. Response time depending on the real putting distance under full (F-RCHB) and incomplete (I-RCHB) vision condition (Mean \pm SD).

\begin{tabular}{|l|l|l|l|l|l|l|}
\hline & \multicolumn{6}{|c|}{ Response time $[\mathbf{s}]$} \\
Condition & $\mathbf{1 . 5} \mathbf{~ m}$ & $\mathbf{2 . 0} \mathbf{~ m}$ & $\mathbf{2 . 5} \mathbf{~ m}$ & $\mathbf{3 . 0} \mathbf{~ m}$ & $\mathbf{3 . 5} \mathbf{~ m}$ & $\mathbf{4 . 0} \mathbf{~ m}$ \\
\hline I-RCHB & $5.70 \pm 5.87$ & $4.47 \pm 2.97$ & $5.61 \pm 5.80$ & $5.77 \pm 4.55$ & $4.99 \pm 4.25$ & $5.33 \pm 4.56$ \\
\hline F-RCHB & $4.64 \pm 4.54$ & $4.35 \pm 4.33$ & $4.17 \pm 4.29$ & $5.13 \pm 5.68$ & $4.61 \pm 4.21$ & $3.38 \pm 3.45$ \\
\hline
\end{tabular}


Table 11. Predicted distance under the I-RCHB, F-RCHB, F-RHC, and F-B conditions (Mean \pm SD).

\begin{tabular}{|l|l|l|l|}
\hline & \multicolumn{3}{|c|}{ Predicted distance $[\mathbf{m}]$} \\
Condition & $\mathbf{2 . 0} \mathbf{~ m}$ & $\mathbf{3 . 0} \mathbf{~ m}$ & $\mathbf{4 . 0} \mathbf{~ m}$ \\
\hline I-RCHB & $2.82 \pm 1.12$ & $2.93 \pm 1.15$ & $3.22 \pm 1.07$ \\
\hline F-RCHB & $3.12 \pm 0.97$ & $3.55 \pm 0.94$ & $4.24 \pm 1.04$ \\
\hline F-RCH & $2.89 \pm 0.95$ & $3.44 \pm 0.98$ & $3.94 \pm 1.15$ \\
\hline F-B & $3.07 \pm 0.86$ & $3.71 \pm 1.01$ & $4.34 \pm 1.14$ \\
\hline
\end{tabular}

Table 12. Follow-up analyses (Wilcoxon test and Bonferroni correction) of the interaction of putting distance and vision condition for predicted putt length at the real putting distance.

\begin{tabular}{|l|l|l|l|l|l|l|l|}
\hline \multicolumn{2}{|c|}{} & $\begin{array}{l}\text { I-RCHB } \\
\text { vs. } \\
\text { F-RCHB }\end{array}$ & $\begin{array}{l}\text { I-RCHB } \\
\text { vs. } \\
\text { F-RCH }\end{array}$ & $\begin{array}{l}\text { I-RCHB } \\
\text { vs. } \\
\text { F-B }\end{array}$ & $\begin{array}{l}\text { F-RCHB } \\
\text { vs. } \\
\text { F-RCH }\end{array}$ & $\begin{array}{l}\text { F-RCHB } \\
\text { vs. } \\
\text { F-B }\end{array}$ & $\begin{array}{l}\text { F-HCB } \\
\text { vs. } \\
\text { F-B }\end{array}$ \\
\hline \multirow{3}{*}{$2.0 \mathrm{~m}$} & $\mathrm{~N}$ & 19 & 19 & 19 & 19 & 19 & 19 \\
\cline { 2 - 8 } & $\mathrm{Z}$ & 1.650 & -.322 & -1.368 & -1.408 & -.724 & -1.127 \\
\cline { 2 - 8 } & $2 \mathrm{p}$ & .099 & .748 & .171 & .159 & .469 & .260 \\
\hline \multirow{3}{*}{$3.0 \mathrm{~m}$} & $\mathrm{~N}$ & 19 & 19 & 19 & 19 & 19 & 19 \\
\cline { 2 - 8 } & $\mathrm{Z}$ & 2.495 & -2.656 & -3.179 & -.483 & -1.569 & -1.288 \\
\cline { 2 - 8 } & $2 \mathrm{p}$ & .013 & $.008^{*}$ & $.001^{*}$ & .629 & .117 & .198 \\
\hline \multirow{3}{*}{$4.0 \mathrm{~m}$} & $\mathrm{~N}$ & 19 & 19 & 19 & 19 & 19 & 19 \\
\cline { 2 - 8 } & $\mathrm{Z}$ & 3.622 & -3.300 & -3.421 & -1.569 & -.262 & -1.569 \\
\cline { 2 - 8 } & $2 \mathrm{p}$ & $<.001^{*}$ & $.001^{*}$ & $.001^{*}$ & .117 & .794 & .117 \\
\hline
\end{tabular}

* Significant after Bonferroni correction. Level of significance $p<.008 \overline{3}$.

Table 13. Constant error of predicted distance under the I-RCHB, F-RCHB, F-RCH, and F-B conditions (Mean \pm SD).

\begin{tabular}{|l|l|l|l|}
\hline & \multicolumn{3}{|c|}{ CE predicted distance [m] } \\
Condition & $\mathbf{2 . 0} \mathbf{~ m}$ & $\mathbf{3 . 0} \mathbf{~ m}$ & $\mathbf{4 . 0} \mathbf{~ m}$ \\
\hline I-RCHB & $0.82 \pm 1.15$ & $-0.07 \pm 1.15$ & $-0.78 \pm 1.07$ \\
\hline F-RCHB & $1.12 \pm 0.97$ & $0.55 \pm 0.94$ & $0.24 \pm 1.04$ \\
\hline F-RCH & $0.89 \pm 0.95$ & $0.44 \pm 0.98$ & $-0.06 \pm 1.04$ \\
\hline F-B & $1.07 \pm 0.86$ & $0.71 \pm 1.01$ & $0.34 \pm 1.14$ \\
\hline
\end{tabular}

Table 14. Follow-up analyses (Wilcoxon test and Bonferroni correction) of the interaction of putting distance and vision condition for the constant error of predicted putt length at the real putting distance.

\begin{tabular}{|l|l|l|l|l|l|l|l|}
\hline \multicolumn{2}{|c|}{} & $\begin{array}{l}\text { I-RCHB } \\
\text { vs. } \\
\text { F-RCHB }\end{array}$ & $\begin{array}{l}\text { I-RCHB } \\
\text { vs. } \\
\text { F-RCH }\end{array}$ & $\begin{array}{l}\text { I-RCHB } \\
\text { vs. } \\
\text { F-B }\end{array}$ & $\begin{array}{l}\text { F-RCHB } \\
\text { vs. } \\
\text { F-RCH }\end{array}$ & $\begin{array}{l}\text { F-RCHB } \\
\text { vs. } \\
\text { F-B }\end{array}$ & $\begin{array}{l}\text { F-HCB } \\
\text { vs. } \\
\text { F-B }\end{array}$ \\
\hline \multirow{3}{*}{$2.0 \mathrm{~m}$} & $\mathrm{~N}$ & 19 & 19 & 19 & 19 & 19 & 19 \\
\cline { 2 - 8 } & $\mathrm{Z}$ & 1.650 & -.322 & -1.368 & -1.408 & -.724 & -1.127 \\
\cline { 2 - 8 } & $2 \mathrm{p}$ & .099 & .748 & .171 & .159 & .469 & .260 \\
\hline \multirow{3}{*}{$3.0 \mathrm{~m}$} & $\mathrm{~N}$ & 19 & 19 & 19 & 19 & 19 & 19 \\
\cline { 2 - 8 } & $\mathrm{Z}$ & 2.495 & -2.656 & -3.179 & -.483 & -1.569 & -1.288 \\
\cline { 2 - 8 } & $2 \mathrm{p}$ & .013 & $.008^{*}$ & $.001^{*}$ & .629 & .117 & .198 \\
\hline \multirow{3}{*}{$4.0 \mathrm{~m}$} & $\mathrm{~N}$ & 19 & 19 & 19 & 19 & 19 & 19 \\
\cline { 2 - 8 } & $\mathrm{Z}$ & 3.622 & -3.300 & -3.421 & -1.569 & -.262 & -1.569 \\
\cline { 2 - 8 } & $2 \mathrm{p}$ & $<.001^{*}$ & $.001^{*}$ & $.001^{*}$ & .117 & .794 & .117 \\
\hline
\end{tabular}

* Significant after Bonferroni correction. Level of significance $p<.008 \overline{3}$. 
Table 15. Variable error of predicted distance under the I-RCHB, F-RCHB, F-RCH, and F-B conditions (Mean \pm SD).

\begin{tabular}{|l|l|l|l|}
\hline & \multicolumn{3}{|c|}{ VE predicted distance $[\mathbf{m}]$} \\
Condition & $\mathbf{2 . 0} \mathbf{~ m}$ & $\mathbf{3 . 0} \mathbf{~ m}$ & $\mathbf{4 . 0} \mathbf{~ m}$ \\
\hline I-RCHB & $0.60 \pm 0.26$ & $0.55 \pm 0.38$ & $0.65 \pm 0.35$ \\
\hline F-RCHB & $0.59 \pm 0.32$ & $0.60 \pm 0.36$ & $0.70 \pm 0.44$ \\
\hline F-RCH & $0.53 \pm 0.28$ & $0.67 \pm 0.40$ & $0.70 \pm 0.30$ \\
\hline F-B & $0.67 \pm 0.31$ & $0.77 \pm 0.32$ & $0.73 \pm 0.36$ \\
\hline
\end{tabular}

Table 16. Results of the two-factor ANOVA with repeated measures (3 distances; 4 vision conditions) for the variable error of predicted putt length. Corrected by Greenhouse-Geisser $\epsilon$.

\begin{tabular}{|l|l|l|l|l|}
\hline Factor & df1 & df2 & F & p \\
\hline Vision condition & 2.54 & 45.74 & 1.35 & .271 \\
\hline Distance & 2.0 & 35.93 & 1.75 & .188 \\
\hline Vision condition $x$ distance & 3.14 & 56.53 & 0.38 & .779 \\
\hline
\end{tabular}

Table 17. Absolute error of the predicted distance under the I-RCHB, F-RCHB, F-RCH, and F-B conditions (Mean \pm SD).

\begin{tabular}{|l|l|l|l|}
\hline & \multicolumn{3}{|c|}{ AE predicted distance $[\mathbf{m}]$} \\
Condition & $\mathbf{2 . 0} \mathbf{~ m}$ & $\mathbf{3 . 0} \mathbf{~ m}$ & $\mathbf{4 . 0} \mathbf{~ m}$ \\
\hline I-RCHB & $1.12 \pm 0.82$ & $0.92 \pm 0.68$ & $1.07 \pm 0.78$ \\
\hline F-RCHB & $1.22 \pm 0.83$ & $0.88 \pm 0.64$ & $0.87 \pm 0.60$ \\
\hline F-RCH & $1.06 \pm 0.74$ & $0.85 \pm 0.65$ & $0.95 \pm 0.64$ \\
\hline F-B & $1.16 \pm 0.74$ & $0.99 \pm 0.74$ & $1.01 \pm 0.63$ \\
\hline
\end{tabular}

Table 18. Results of the two-factor ANOVA with repeated measures (3 distances; 4 vision conditions) for absolute error of the predicted putt length. Corrected by Greenhouse-Geisser $\epsilon$.

\begin{tabular}{|l|l|l|l|l|}
\hline Factor & df1 & df2 & F & p \\
\hline Vision condition & 2.36 & 42.39 & 0.620 & .568 \\
\hline Distance & 1.42 & 25.48 & 2.77 & .097 \\
\hline Vision condition $x$ distance & 3.66 & 65.95 & 0.71 & .574 \\
\hline
\end{tabular}

Table 19. Confidene of prediction under the I-RCHB, F-RCHB, F-RCH, and F-B conditions (Mean \pm SD).

\begin{tabular}{|l|l|l|l|}
\hline & \multicolumn{3}{|c|}{ Confidence $[\mathbf{1 - 5}]$} \\
Condition & $\mathbf{2 . 0} \mathbf{~ m}$ & $\mathbf{3 . 0} \mathbf{~ m}$ & $\mathbf{4 . 0} \mathbf{~ m}$ \\
\hline I-RCHB & $2.92 \pm 0.67$ & $2.92 \pm 0.80$ & $2.82 \pm 0.87$ \\
\hline F-RCHB & $3.38 \pm 0.75$ & $3.28 \pm 0.78$ & $3.39 \pm 0.71$ \\
\hline F-RCH & $3.03 \pm 0.83$ & $3.13 \pm 0.78$ & $3.29 \pm 0.78$ \\
\hline F-B & $3.21 \pm 0.72$ & $3.30 \pm 0.80$ & $3.33 \pm 0.81$ \\
\hline
\end{tabular}

Table 20. Response time depending on the real putting distance under the I-RCHB, F-RCHB, F-RCH, and F-B condition (Mean \pm SD).

\begin{tabular}{|l|l|l|l|}
\hline & \multicolumn{3}{|c|}{ Response time [s] } \\
Condition & $\mathbf{2 . 0} \mathbf{~ m}$ & $\mathbf{3 . 0} \mathbf{~ m}$ & $\mathbf{4 . 0} \mathbf{~ m}$ \\
\hline I-RCHB & $4.73 \pm 3.01$ & $5.14 \pm 3.34$ & $5.92 \pm 7.11$ \\
\hline F-RCHB & $5.45 \pm 4.73$ & $5.45 \pm 6.69$ & $4.57 \pm 4.59$ \\
\hline F-RCH & $5.43 \pm 4.71$ & $5.02 \pm 3.49$ & $4.85 \pm 5.97$ \\
\hline F-B & $4.07 \pm 2.10$ & $4.62 \pm 2.75$ & $5.15 \pm 5.20$ \\
\hline
\end{tabular}

